\title{
Application of Clifford Algebra in Solving the Eigen Equations of Quantum Mechanics
}

\author{
Ying-Qiu Gu \\ School of Mathematical Science, Fudan University, Shanghai 200433, China*
}

Clifford algebra is unified language and efficient tool for geometry and physics. In this paper, we introduce this algebra to derive the integrable conditions for Dirac and Pauli equations. This algebra shows the standard operation procedure and deep insights into the structure of the equations. Usually, the integrable condition is related to the special symmetry of transformation group, which involves some advanced mathematical tools and its availability is limited. In this paper, the integrable conditions are only regarded as algebraic conditions. The commutators expressed by Clifford algebra have a neat and covariant form, which are naturally valid in curvilinear coordinate system and curved space-time. For Pauli and Schrödinger equation, many solutions in axisymmetric potential and magnetic field are also integrable. We get the scalar eigen equation in dipole magnetic field. By the virtue of Clifford algebra, the physical researches may be greatly promoted.

Keywords: Clifford algebra; Abelian Lie algebra; eigen function; separation of variables; Dirac equation; Pauli equation, dipole magnetic field; axisymmetric potential.

PACS numbers: 02.30.Ik, 02.30.Jr, 03.65.-w, 11.30.-j

\section{Contents}

I. Introduction

II. The Equation Represented by Clifford Algebra

III. Integrable Conditions for Dirac Equations

${ }^{*}$ Electronic address: yqgu@fudan.edu.cn 


\section{INTRODUCTION}

In quantum mechanics, we have the following important theorem for solving the rigorous eigen solution of a Hamiltonian[1].

Theorem 1 For two linear Hermitian operators $\hat{H}_{1}, \hat{H}_{2}$ on a vector space, if they commute with each other $\left[\hat{H}_{1}, \hat{H}_{2}\right]=0$, then they have common eigen vectors.

This theorem is the foundation of method of separating variables. For a Hamiltonian operator $\hat{H}$, if we can construct the following Hermitian operators chain[2],

$$
\hat{H}_{1}=H_{1}\left(\partial_{1}\right), \quad \hat{H}_{2}=H_{2}\left(\partial_{1}, \partial_{2}\right), \quad \cdots, \quad \hat{H}=H_{n}\left(\partial_{1}, \partial_{2}, \cdots, \partial_{n}\right)
$$

which form an Abelian Lie algebra

$$
\left[\hat{H}_{j}, \hat{H}_{k}\right]=0, \quad(j, k=1, \cdots, n),
$$

then (1.2) forms the integrable conditions of the eigen equation $\hat{H} \phi=E \phi$, and the eigen solutions can be completely solved by means of separating variables.

In $3+1$ Minkowski space-time, to solve eigenfunctions of the linear Dirac equation with central potential, we have the commutative relations[3]

$$
\left[\hat{H}, \hat{J}_{z}\right]=[\hat{H}, \hat{K}]=\left[\hat{K}, \hat{J}_{z}\right]=0
$$

where the commutators $\left(\hat{J}_{z}, \hat{K}, \hat{H}\right)$ are respectively angular momentum, spin-orbit coupling and total energy operators defined by

$$
\hat{J}_{z}\left(\partial_{\varphi}\right)=-i \hbar \partial_{\varphi}+\frac{1}{2} \hbar S_{3}, \quad \hat{K}\left(\partial_{\theta}, \partial_{\varphi}\right)=\gamma^{0}(\hat{L} \cdot \vec{S}+\hbar), \quad \hat{H}=H\left(\partial_{\varphi}, \partial_{\theta}, \partial_{r}\right) .
$$

The commutators $\left(\hat{J}_{z}, \hat{K}, \hat{H}\right)$ form the complete chain of operators (1.3), which enable us to solve the eigen solutions by separation of variables, and then the original problem reduces to ordinary differential equations. In some cases with special potentials, the eigen solutions of Dirac equation can be solved similarly[4]-[8]. So the existence of complete commutative operator chain is the sufficient condition of integrability for Eigen equations in mechanics, such Dirac equation and Schrödinger equation.

By (1.4) we find the commutative operators are usually related to some symmetries of the system which can be only displayed in a curvilinear coordinate system. For such problem, it will be very convenient to use Clifford algebra for discussion and calculation. Clifford algebra is a unified 
language for physics, which faithfully reflects the intrinsic symmetry and property of space-time and fields[9-14]. We can exactly express dynamical equations in physics by Clifford algebra, and in the representation there is nothing superfluous or missed. The parameters in Clifford algebra all have clear physical meanings and the operations become simple and standard. In contrast with the conventional and direct calculation in [2], we can deeply realize the superiority of Clifford algebra.

\section{THE EQUATION REPRESENTED BY CLIFFORD ALGEBRA}

There are several definitions for Clifford algebra[15, 16]. Clifford algebra is also called geometric algebra. If the definition is directly related to geometric concepts, it will bring great convenience to the study and research of geometry[17].

Definition 1 Assume the element of an $n=p+q$ dimensional space-time $\mathbb{M}^{p, q}$ over $\mathbb{R}$ is given by

$$
d \mathbf{x}=\gamma_{\mu} d x^{\mu}=\gamma^{\mu} d x_{\mu}=\gamma_{a} \delta X^{a}=\gamma^{a} \delta X_{a}
$$

where $\gamma_{a}$ is the local orthogonal frame and $\gamma^{a}$ the coframe. The space-time is endowed with distance $d s=|d \mathbf{x}|$ and oriented volumes $d V_{k}$ calculated by

$$
\begin{aligned}
& d \mathbf{x}^{2}=\frac{1}{2}\left(\gamma_{\mu} \gamma_{\nu}+\gamma_{\nu} \gamma_{\mu}\right) d x^{\mu} d x^{\nu}=g_{\mu \nu} d x^{\mu} d x^{\nu}=\eta_{a b} \delta X^{a} \delta X^{b} \\
& d V_{k}=d \mathbf{x}_{1} \wedge d \mathbf{x}_{2} \wedge \cdots \wedge d \mathbf{x}_{k}=\gamma_{\mu \nu \cdots \omega} d x_{1}^{\mu} d x_{2}^{\nu} \cdots d x_{k}^{\omega}, \quad(1 \leq k \leq n)
\end{aligned}
$$

in which the Minkowski metric in matrix form reads $\left(\eta_{a b}\right)=\operatorname{diag}\left(I_{p},-I_{q}\right)$, and Grassmann basis $\gamma_{\mu \nu \cdots \omega}=\gamma_{\mu} \wedge \gamma_{\nu} \wedge \cdots \wedge \gamma_{\omega} \in \Lambda^{k} \mathbb{M}^{p, q}$. Then the following number with basis

$$
C=c_{0} I+c_{\mu} \gamma^{\mu}+c_{\mu \nu} \gamma^{\mu \nu}+\cdots+c_{12 \cdots n} \gamma^{12 \cdots n}, \quad\left(\forall c_{k} \in \mathbb{R}\right)
$$

together with multiplication rule of basis given in (2.2) and associativity define the $2^{n}$-dimensional real universal Clifford algebra $C \ell_{p, q}$.

For clearness, it is necessary to clarify the conventions and notations frequently used in the paper. We take $c=1$ as unit of speed. The Einstein's summation is used. For index, we use the Greek characters for curvilinear coordinates, Latin characters for local Minkowski coordinates and $\{i, j, k, l\}$ for spatial indices. The Pauli matrices are expressed by

$$
\begin{aligned}
& \sigma^{a}=\left\{\left(\begin{array}{ll}
1 & 0 \\
0 & 1
\end{array}\right),\left(\begin{array}{ll}
0 & 1 \\
1 & 0
\end{array}\right),\left(\begin{array}{cc}
0 & -i \\
i & 0
\end{array}\right),\left(\begin{array}{cc}
1 & 0 \\
0 & -1
\end{array}\right)\right\}, \\
& \tilde{\sigma}^{a}=\left(\sigma^{0},-\sigma^{1},-\sigma^{2},-\sigma^{3}\right), \quad \sigma^{\mu}=f_{a}^{\mu} \sigma^{a}, \quad \widetilde{\sigma}_{\mu}=f_{\mu}{ }^{a} \widetilde{\sigma}_{a},
\end{aligned}
$$


where $a, \mu \in\{0,1,2,3\}, f_{a}^{\mu}, f_{\mu}^{a}$ are the frame coefficients satisfying

$$
f_{\mu}^{a} f_{\nu}^{b} \eta_{a b}=g_{\mu \nu}, \quad f_{a}^{\mu} f_{b}^{\nu} \eta_{a b}=g^{\mu \nu}, \quad \eta_{a b}=\operatorname{diag}(1,-1,-1,-1)
$$

For frame coefficient, the first index is always for curvilinear coordinate, and the second index for Minkowski index. In $1+3$ dimensional space-time, $\gamma^{a}$ and $\gamma^{\mu}$ can be expressed by Dirac matrices

$$
\gamma^{a}=\left(\begin{array}{cc}
0 & \widetilde{\sigma}^{a} \\
\sigma^{a} & 0
\end{array}\right), \quad \gamma^{5}=\left(\begin{array}{cc}
I & 0 \\
0 & -I
\end{array}\right), \quad \vartheta^{5}=\left(\begin{array}{cc}
0 & -i I \\
i I & 0
\end{array}\right) .
$$

In equivalent sense, $\gamma^{a}$ forms the unique representation for generators of $C \ell_{1,3}$. In curved spacetime $\gamma^{\mu}=f_{a}^{\mu} \gamma^{a}$ and $\gamma_{\mu}=f_{\mu}{ }^{a} \gamma_{a}$. Since the Clifford algebra is isomorphic to matrix algebra[15-17], we need not distinguish matrix $\gamma^{a}$ with tetrad $\gamma^{a}$. Thus we have

$$
\gamma_{a} \gamma_{b}+\gamma_{b} \gamma_{a}=2 \eta_{a b}, \quad \gamma_{\mu} \gamma_{\nu}+\gamma_{\nu} \gamma_{\mu}=2 g_{\mu \nu}
$$

In (2.9), the product between basis such as $\gamma_{a} \gamma_{b}$ is called Clifford product, which is isomorphic to matrix product. For Clifford product, we have[18]

Theorem 2 For basis of Clifford algebra, we have the following relations

$$
\begin{gathered}
\gamma^{\mu} \gamma^{\theta_{1} \theta_{2} \cdots \theta_{k}}=\gamma^{\mu} \odot \gamma^{\theta_{1} \theta_{2} \cdots \theta_{k}}+\gamma^{\mu \theta_{1} \cdots \theta_{k}} \\
\gamma^{\theta_{1} \theta_{2} \cdots \theta_{k}} \gamma^{\mu}=\gamma^{\theta_{1} \theta_{2} \cdots \theta_{k}} \odot \gamma^{\mu}+\gamma^{\theta_{1} \cdots \theta_{k} \mu} . \\
\gamma^{\lambda_{1} \lambda_{2} \cdots \lambda_{k}} \gamma^{\theta_{1} \theta_{2} \cdots \theta_{l}}=\sum_{n=1}^{\min (k, l)} \gamma^{\lambda_{1} \lambda_{2} \cdots \lambda_{k}} \odot^{n} \gamma^{\theta_{1} \theta_{2} \cdots \theta_{l}}+\gamma^{\lambda_{1} \lambda_{2} \cdots \lambda_{k} \theta_{1} \theta_{2} \cdots \theta_{l}} \\
\gamma_{a_{1} a_{2} \cdots a_{n-1}}=\epsilon_{a_{1} a_{2} \cdots a_{n}} \gamma_{12 \cdots n} \gamma^{a_{n}} \\
\gamma_{a_{1} a_{2} \cdots a_{n-k}}=\frac{1}{k !} \epsilon_{a_{1} a_{2} \cdots a_{n}} \gamma_{12 \cdots n} \gamma^{a_{n-k+1} \cdots a_{n}} .
\end{gathered}
$$

In which $\odot^{k}$ is multi-inner product of Clifford algebra. For example,

$$
\begin{gathered}
\gamma^{\mu} \odot \gamma^{\nu}=\gamma^{\mu} \cdot \gamma^{\nu}=g^{\mu \nu}, \quad \gamma^{\mu \nu} \odot^{2} \gamma^{\alpha \beta}=g^{\mu \beta} g^{\nu \alpha}-g^{\mu \alpha} g^{\nu \beta} \\
\gamma^{\mu} \odot \gamma^{\theta_{1} \theta_{2} \cdots \theta_{k}}=g^{\mu \theta_{1}} \gamma^{\theta_{2} \cdots \theta_{k}}-g^{\mu \theta_{2}} \gamma^{\theta_{1} \theta_{3} \cdots \theta_{k}}+\cdots+(-1)^{k+1} g^{\mu \theta_{k}} \gamma^{\theta_{1} \cdots \theta_{k-1}}, \\
\gamma^{\theta_{1} \theta_{2} \cdots \theta_{k}} \odot \gamma^{\mu}=(-1)^{k+1} g^{\mu \theta_{1}} \gamma^{\theta_{2} \cdots \theta_{k}}+(-1)^{k} g^{\mu \theta_{2}} \gamma^{\theta_{1} \theta_{3} \cdots \theta_{k}}+\cdots+g^{\mu \theta_{k}} \gamma^{\theta_{1} \cdots \theta_{k-1}} . \\
\gamma^{\mu \nu} \odot \gamma^{\alpha \beta}=g^{\mu \beta} \gamma^{\nu \alpha}-g^{\mu \alpha} \gamma^{\nu \beta}+g^{\nu \alpha} \gamma^{\mu \beta}-g^{\nu \beta} \gamma^{\mu \alpha} .
\end{gathered}
$$

In the curvilinear coordinate system, Dirac equation with electromagnetic potential and nonlinear potential is given by $[13,14,19,20]$

$$
\gamma^{\mu}\left(\hat{p}_{\mu}+\frac{1}{2} \hbar \gamma^{5} \Omega_{\mu}\right) \phi+i \gamma^{5} F_{\vartheta} \phi=\left(m c-F_{\gamma}\right) \phi,
$$


in which the parameters and operators are defined as

$$
\begin{aligned}
\hat{p}_{\mu} & =i \hbar\left(\partial_{\mu}+\Upsilon_{\mu}\right)-e A_{\mu}, \quad \Upsilon_{\mu}=\frac{1}{2} f_{a}^{\nu}\left(\partial_{\mu} f_{\nu}{ }^{a}-\partial_{\nu} f_{\mu}{ }^{a}\right), \\
\Omega^{\alpha} & =-\frac{1}{2}\left(\epsilon^{d a b c} f_{d}^{\alpha} f_{a}^{\mu} f_{b}^{\nu}\right) \partial_{\mu} f_{\nu}{ }^{e} \eta_{c e}=\frac{1}{4 \sqrt{g}} \epsilon^{\alpha \mu \nu \omega} \eta_{a b} f_{\omega}{ }^{a}\left(\partial_{\nu} f_{\mu}{ }^{b}-\partial_{\mu} f_{\nu}{ }^{b}\right) .
\end{aligned}
$$

$\Upsilon_{\mu}$ is the Keller connection and $\Omega_{\mu}$ is Gu-Nester potential. If the metric can be diagonalized, we have $\Omega_{\mu} \equiv 0$. The nonlinear potential $F=F(\check{\gamma}, \check{\vartheta})$ is a function of quadratic scalar and pseudo scalar,

$$
\check{\gamma}=\phi^{+} \gamma^{0} \phi, \quad \check{\vartheta}=\phi^{+} \vartheta^{5} \phi, \quad F_{\gamma}=\frac{\partial F}{\partial \check{\gamma}}, \quad F_{\vartheta}=\frac{\partial F}{\partial \check{\vartheta}} .
$$

the coefficients in (2.19) belong to $\Lambda^{0} \cup \Lambda^{1} \cup \Lambda^{3} \cup \Lambda^{4} \subset C \ell_{1,3}$.

To get the the Hamiltonian formalism of (2.19), the time $t$ should be global cosmic time. That is, the Hamiltonian formalism can be clearly expressed only in the Gu's natural coordinate system(NCS) with metric $d s^{2}=g_{00} d t^{2}-g_{k l} d x^{k} d x^{l}$, in which $d \tau=\sqrt{g_{00}} d t$ defines the Newton's realistic cosmic time[21]. In NCS, to lift and lower the index of a vector means

$$
\Upsilon^{0}=g^{00} \Upsilon_{0}, \quad \Upsilon^{k}=-g^{k l} \Upsilon_{l}, \quad g_{k l} g^{l n}=\delta_{k}^{n}
$$

Then the eigen equation of (2.19) can be rewritten in the following Hamiltonian formalism

$$
i \alpha^{0}\left(\partial_{t}+\Upsilon_{t}\right) \phi=\hat{H} \phi, \quad \hat{H}=-\alpha^{k} \hat{p}_{k}+e A_{0} \alpha^{0}+\left(m c-F_{\gamma}\right) \gamma^{0}-F_{\vartheta} \vartheta^{5}-\Omega_{\mu} \hat{S}^{\mu},
$$

where $\hat{H}$ is the Hamiltonian in curved space-time, and $\left(\alpha^{\mu}, \hat{S}^{\mu}\right)$ are respectively current and spin operators defined by

$$
\alpha^{\mu} \equiv \gamma^{0} \gamma^{\mu}=\operatorname{diag}\left(\sigma^{\mu}, \widetilde{\sigma}^{\mu}\right), \quad \hat{S}^{\mu} \equiv \frac{1}{2} \hbar \alpha^{\mu} \gamma^{5}=\frac{1}{2} \hbar \operatorname{diag}\left(\sigma^{\mu},-\widetilde{\sigma}^{\mu}\right) .
$$

$\vec{S} \in \Lambda^{3}$ is the usual spin of Dirac bispinor.

To get the eigen state of energy of a particle, we must separate the drifting motion of the particle by a local Lorentz transformation to its comoving coordinate system. Then we get eigen state

$$
\hat{H} \phi=i \alpha^{0}\left(\partial_{t}+\Upsilon_{t}\right) \phi=E \phi, \quad E \in \mathbb{R}
$$

In this case of the spinor at energy eigen state, the Hamiltonian $\hat{H}$ in (2.26) has the following properties: $1^{\circ} \hat{H}$ is Hermitian, so its eigen values are all real. $2^{\circ} \hat{H}$ is a linear operator with respect to derivatives $\nabla_{k}=\partial_{k}+\Upsilon_{k} .3^{\circ}$ Formally, in (2.26) $\hat{H} \in C \ell_{4,0}$ with the generators $\left(\gamma^{0}, \alpha^{k}\right)$. We introduce the generators of $C \ell_{4,0}$ as follows,

$$
\begin{gathered}
\vartheta^{a}=\left(\gamma^{0}, \alpha^{k}\right), \quad \vartheta_{a}=\delta_{a b} \vartheta^{b}, \quad \vartheta^{a} \vartheta^{b}+\vartheta^{b} \vartheta^{a}=2 \delta^{a b}, \\
\vartheta^{\mu}=t_{a}^{\mu} \vartheta^{a}, \quad \vartheta_{\mu}=t_{\mu}^{a} \vartheta_{a}, \quad \vartheta_{\mu} \vartheta_{\nu}+\vartheta_{\nu} \vartheta_{\mu}=2 g_{\mu \nu}=2 \operatorname{diag}\left(1, g_{k l}\right), \\
t_{\mu}^{a} t_{\nu}^{b} \delta_{a b}=g_{\mu \nu}, \quad t_{\mu}{ }_{\mu} t_{b}^{\mu}=\delta_{b}^{a}, \quad t_{\mu}^{a} t^{\nu}{ }_{a}=\delta_{\mu}^{\nu} .
\end{gathered}
$$


The complete bases of $C \ell_{4,0}$ and their relations are given by

$$
I, \quad \vartheta^{a}, \quad \vartheta^{a b} \equiv \vartheta^{a} \wedge \vartheta^{b}=-\frac{1}{2} \epsilon^{a b c d} \vartheta_{c d} \vartheta^{5}, \quad \vartheta^{a b c}=-\epsilon^{a b c d} \vartheta_{d} \vartheta^{5}, \quad \vartheta^{0123}=\vartheta^{5}
$$

By (2.27)-(2.30), the Hamiltonian can be rewritten in Clifford algebra $C \ell_{4,0}$ as

$$
\hat{H}=e A_{0} \sqrt{g^{00}}+\vartheta^{\mu} \hat{P}_{\mu}+\frac{i \hbar}{2} \Omega_{\mu} \vartheta^{0 \mu} \vartheta^{5}+\frac{i \hbar}{2} \sqrt{g^{00}} \Omega_{0} \vartheta^{0} \vartheta^{5}-F_{\vartheta} \vartheta^{5},
$$

in which the Clifford numbers

$$
A_{0} \in \Lambda^{0}, \quad \hat{P}_{\mu} \in \Lambda^{1}, \quad \vec{\Omega} \in \Lambda^{2}, \quad \Omega_{0} \in \Lambda^{3}, \quad F_{\vartheta} \in \Lambda^{4}
$$

We find the grade of some quantities in hyperbolic system $\gamma^{\mu}$ have been changed in elliptic system $\vartheta^{\mu}$. For example, $(\vec{\Omega}, \vec{S}) \in \Lambda^{3}$ in system $\gamma^{\mu}$ have been converted into $(\vec{\Omega}, \vec{S}) \in \Lambda^{2}$ in system $\vartheta^{\mu}$. The 4-vector momentum $\hat{P}_{\mu}$ is redefined by

$$
\hat{P}_{\mu}=\left(m c-F_{\gamma},-i \hbar\left(\partial_{k}+\Upsilon_{k}\right)-e A_{k}\right)
$$

In $\hat{P}_{\mu}$ the sign of $e A_{k}$ should be changed, this is because the Minkowski metric $\eta^{k k}=-1$ in $C \ell_{1,3}$ has been converted into $\delta^{k k}=1$ in $C \ell_{4,0}$. So we have $\hat{P}^{k} \phi=\hat{P}_{k} \phi \rightarrow m v_{k} \phi$.

In order to calculate the derivatives of Clifford numbers, we introduce the following Christoffellike connections

$$
\Theta_{\alpha \beta}^{\mu}=t^{\mu}{ }_{a} \partial_{\alpha} t_{\beta}^{a}
$$

We have relations

$$
\Gamma_{\mu \alpha}^{\alpha}=\Theta_{\mu \alpha}^{\alpha}, \quad \Upsilon_{\mu}=\frac{1}{2}\left(\Theta_{\mu \alpha}^{\alpha}-\Theta_{\alpha \mu}^{\alpha}\right)
$$

For tetrad the derivatives are given by

$$
\begin{aligned}
& \partial_{\alpha} \vartheta_{\beta}=\vartheta_{a} \partial_{\alpha} t_{\beta}^{a}=\vartheta_{\mu} t_{a}^{\mu} \partial_{\alpha} t_{\beta}^{a}=\vartheta_{\mu} \Theta_{\alpha \beta}^{\mu}, \\
& \partial_{\alpha} \vartheta^{\mu}=\vartheta^{a} \partial_{\alpha} t^{\mu}{ }_{a}=\vartheta^{\beta} t_{\beta}{ }^{a} \partial_{\alpha} t^{\mu}{ }_{a}=-\vartheta^{\beta} \Theta_{\alpha \beta}^{\mu} .
\end{aligned}
$$

So we can define the frame covariant derivatives $\bar{\nabla}_{\alpha}$ for Clifford numbers as

$$
\begin{aligned}
\partial_{\alpha} \mathbf{A} & =\partial_{\alpha}\left(A^{\mu} \vartheta_{\mu}\right)=\vartheta_{\mu} \bar{\nabla}_{\alpha} A^{\mu}=\vartheta_{\mu}\left(\partial_{\alpha} A^{\mu}+\Theta_{\alpha \beta}^{\mu} A^{\beta}\right) \\
& =\partial_{\alpha}\left(A_{\mu} \vartheta^{\mu}\right)=\vartheta^{\mu} \bar{\nabla}_{\alpha} A_{\mu}=\vartheta^{\mu}\left(\partial_{\alpha} A_{\mu}-\Theta_{\alpha \mu}^{\beta} A_{\beta}\right), \\
\partial_{\alpha} \mathbf{N} & =\vartheta_{\mu \nu} \bar{\nabla}_{\alpha} N^{\mu \nu}=\vartheta_{\mu \nu}\left(\partial_{\alpha} N^{\mu \nu}+\Theta_{\alpha \beta}^{\mu} N^{\beta \nu}+\Theta_{\alpha \beta}^{\nu} N^{\mu \beta}\right) \\
& =\vartheta^{\mu \nu} \bar{\nabla}_{\alpha} N_{\mu \nu}=\vartheta^{\mu \nu}\left(\partial_{\alpha} N_{\mu \nu}-\Theta_{\alpha \mu}^{\beta} N_{\beta \nu}-\Theta_{\alpha \nu}^{\beta} N_{\mu \beta}\right),
\end{aligned}
$$


and so on. It should be stressed that, here we have $\partial_{\alpha} \gamma^{a} \equiv 0$, which is only related to algebra calculation. But for the absolute derivative or covariant derivative in differential geometry, we have connection[14]

$$
\mathfrak{d}_{\alpha} \gamma^{a}=-f_{\mu}^{a} \gamma_{; \alpha}^{\mu}=\eta^{a b} \mathfrak{d}_{\alpha} \gamma_{b}, \quad \gamma_{; \alpha}^{\mu} \equiv \partial_{\alpha} \gamma^{\mu}+\Gamma_{\alpha \nu}^{\mu} \gamma^{\nu}
$$

In general case $\gamma_{; \alpha}^{\mu} \neq 0$.

\section{INTEGRABLE CONDITIONS FOR DIRAC EQUATIONS}

Now we take spherical coordinate system as example to show the concepts and the advantage of Clifford algebra. For static potential $A_{0}=A_{0}(r, \theta)$ and magnetic field $\vec{B}=B_{z} \vartheta^{z} \vartheta^{5}$, we have $\vec{A}=A(r, \theta) \vartheta^{\varphi}$ and

$$
\begin{gathered}
\left(g_{\mu \nu}\right)=\operatorname{diag}\left(1,1, r^{2}, r^{2} \sin ^{2} \theta\right), \quad\left(t_{\mu}^{a}\right)=\operatorname{diag}(1,1, r, r \sin \theta) . \\
\Upsilon_{\mu}=\left(0, \frac{1}{r}, \frac{1}{2} \cot \theta, 0\right), \quad \Omega_{\mu}=0 . \\
\hat{P}_{\mu}=\left(m c-F_{\gamma},-i \hbar\left(\partial_{r}+\frac{1}{r}\right),-i \hbar\left(\partial_{\theta}+\frac{1}{2} \cot \theta\right),-i \hbar \partial_{\varphi}-e A\right) .
\end{gathered}
$$

In the case of $\Omega^{\mu}=0$, the Hamiltonian becomes

$$
\hat{H}=e A_{0}+\vartheta^{\mu} \hat{P}_{\mu}-F_{\vartheta} \vartheta^{5}
$$

Then we simply have

$$
\hat{L}_{z}=-i \hbar \partial_{\varphi}, \quad\left[\hat{H}, \hat{L}_{z}\right]=0
$$

Different from (1.4), the spin $\hat{S}$ vanishes in the operator, this is because the spinor $\phi$ has been implicitly made the following spin- $\frac{1}{2}$ transformation[22],

$$
\psi=\Pi \psi^{\prime}, \quad \widetilde{\psi}=\Pi^{*} \widetilde{\psi}^{\prime},
$$

in which

$$
\Pi=\Pi^{*}=\frac{1}{\sqrt{2}}\left(\begin{array}{rr}
i \exp \left[-\frac{i}{2}\left(\theta+\varphi+\frac{\pi}{2}\right)\right], & \exp \left[\frac{i}{2}\left(\theta-\varphi+\frac{\pi}{2}\right)\right] \\
-\exp \left[-\frac{i}{2}\left(\theta-\varphi+\frac{\pi}{2}\right)\right], & -i \exp \left[\frac{i}{2}\left(\theta+\varphi+\frac{\pi}{2}\right)\right]
\end{array}\right)
$$

Now we look for the second Hermitian commutator

$$
\hat{T}\left(\partial_{\theta}, \partial_{\varphi}\right)=T^{0}+T^{\theta} \hat{P}_{\theta}+T^{\varphi} \hat{P}_{\varphi}
$$


in which $\left(T^{0}, T^{\theta}, T^{\varphi}\right)$ are all Hermitian matrices. By $\left[\hat{L}_{z}, \hat{T}\right]=0$, we get $\partial_{\varphi} \hat{T}=0$. This means $\left(T^{0}, T^{\theta}, T^{\varphi}\right)$ should be independent of $\varphi$. We examine $[\hat{H}, \hat{T}]=0$. For any eigen solution $\phi$, we have $\hat{P}_{\varphi} \phi=\left(m_{z} \hbar-e A\right) \phi$. This means $\hat{P}_{\varphi}$ can be regarded as an ordinary function for any eigen solution. Then we have integrable condition as

$$
\begin{aligned}
0 & =[\hat{H}, \hat{T}]=[\hat{H}, \hat{T}]_{A}-i \hbar \vartheta^{\mu} \partial_{\mu} \hat{T}+i \hbar T^{\theta} \partial_{\theta} \hat{H} \\
& =\left[\vartheta^{r}, T^{\theta}\right] \hat{P}_{r} \hat{P}_{\theta}+\left[\vartheta^{\theta}, T^{\theta}\right] \hat{P}_{\theta}^{2}+\left[\vartheta^{r}, \hat{T}\right] \hat{P}_{r}+\cdots
\end{aligned}
$$

where $[\hat{H}, \hat{T}]_{A}$ stands for algebraic commutation taking $\partial_{\mu}$ as ordinary numbers. Then we get integrable conditions

$$
\left[\vartheta^{r}, T^{\theta}\right]=\left[\vartheta^{\theta}, T^{\theta}\right]=\left[\vartheta^{r}, \hat{T}\right]=\cdots=0
$$

By Hermiticity of $\hat{T}$ and (2.30), $T^{\theta}$ can be represented by the following Clifford algebra

$$
T^{\theta}=X+Y_{\mu} \vartheta^{\mu}+i Z_{\mu \nu} \vartheta^{\mu \nu}+i U_{\mu} \vartheta^{\mu} \vartheta^{0 r \theta \varphi}+V \vartheta^{0 r \theta \varphi}
$$

in which all coefficients are real functions of $(r, \theta)$ and $Z_{\mu \nu}=-Z_{\nu \mu}$. Then by Thm.2 and (3.1), we have

$$
\begin{aligned}
\frac{1}{2}\left[\vartheta^{r}, T^{\theta}\right] & =Y_{\mu} \vartheta^{r \mu}+i Z_{\mu \nu} \vartheta^{r} \odot \vartheta^{\mu \nu}+i U_{\mu}\left(\vartheta^{r} \odot \vartheta^{\mu}\right) \vartheta^{0 r \theta \varphi}+V \vartheta^{r} \odot \vartheta^{0 r \theta \varphi} \\
& =Y_{\mu} \vartheta^{r \mu}+2 i Z_{r \nu} g^{r r} \vartheta^{\nu}+i U_{r} g^{r r} \vartheta^{0 r \theta \varphi}-V g^{r r} \vartheta^{0 \theta \varphi}
\end{aligned}
$$

By $\left[\vartheta^{r}, T^{\theta}\right]=0$ we get

$$
\begin{gathered}
Y_{0}=Y_{\theta}=Y_{\varphi}=Z_{0 r}=Z_{r \theta}=Z_{r \varphi}=U_{r}=V=0 . \\
T^{\theta}=X+Y_{r} \vartheta^{r}+i Z_{0 \theta} \vartheta^{0 \theta}+i Z_{0 \varphi} \vartheta^{0 \varphi}+i Z_{\theta \varphi} \vartheta^{\theta \varphi}+i\left(U_{0} \vartheta^{0}+U_{\theta} \vartheta^{\theta}+U_{\varphi} \vartheta^{\varphi}\right) \vartheta^{0 r \theta \varphi} .
\end{gathered}
$$

By $\left[\vartheta^{\theta}, T^{\theta}\right]=0$ we get

$$
\begin{gathered}
\frac{1}{2}\left[\vartheta^{\theta}, T^{\theta}\right]=Y_{r} \vartheta^{\theta r}+2 i\left(-Z_{0 \theta} g^{\theta \theta} \vartheta^{0}-Z_{0 \varphi} \vartheta^{0 \theta \varphi}+Z_{\theta \varphi} g^{\theta \theta} \vartheta^{\varphi}\right)+i U_{\theta} g^{\theta \theta} \vartheta^{0 r \theta \varphi}=0, \\
T^{\theta}=X+i\left(U_{0} \vartheta^{0}+U_{\varphi} \vartheta^{\varphi}\right) \vartheta^{0 r \theta \varphi}=X+i U_{0} \vartheta^{r \theta \varphi}-i U_{\varphi} g^{\varphi \varphi} \vartheta^{0 r \theta} .
\end{gathered}
$$

Similarly, by Clifford calculus of (3.9) we finally derive the integrable conditions as

$$
\begin{gathered}
\hat{T}=\operatorname{ir}\left(\vartheta^{0 r \theta} \hat{P}_{\theta}+\vartheta^{0 r \varphi} \hat{P}_{\varphi}\right) . \\
A_{0}=V(r), \quad A=A(\theta), \quad \partial_{\theta} F_{\gamma}=0, \quad F_{\vartheta}=0 .
\end{gathered}
$$

According to

$$
A=A(\theta), \quad r \vartheta^{0 r \theta}=\vartheta^{012}, \quad r \vartheta^{0 r \varphi}=\frac{1}{\sin \theta} \vartheta^{013}
$$


we find $\hat{T}$ is actually independent of $r$. In Minkowski space-time the condition for vector potential becomes

$$
\vec{A}=\vartheta^{\varphi} A_{\varphi}=\frac{1}{r} U(\theta)(-\sin \varphi, \cos \varphi, 0), \quad U \equiv \frac{A(\theta)}{\sin \theta}
$$

The result is the same as derived in [2].

Since the bispinor $\phi$ always has a spin-axis, we certainly have a commutator $\partial_{\varphi}$ for any eigen state for a single spinor. Now We consider the Dirac equation in coordinate system $(t, r, z, \varphi)$ with metric

$$
d s^{2}=d t^{2}-R^{2} d r^{2}-Z^{2} d z^{2}-\rho^{2} d \varphi^{2}
$$

where $(R, Z, \rho)$ are smooth functions of $(r, z)$. We get the Keller connection as[20]

$$
\sqrt{g}=R Z \rho, \quad \Upsilon_{\mu}=\frac{1}{2}\left(0, \frac{\partial_{r}(Z \rho)}{Z \rho}, \frac{\partial_{z}(R \rho)}{R \rho}, 0\right) .
$$

For vector potential $\vec{A}=A(r, z) \vartheta^{\varphi}$, we have

$$
\begin{gathered}
\hat{H}=e A_{0}+\vartheta^{\mu} \hat{P}_{\mu}-F_{\vartheta} \vartheta^{5} \\
\hat{P}_{\mu}=\left[m c-F_{\gamma},-i \hbar\left(\partial_{r}+\Upsilon_{r}\right),-i \hbar\left(\partial_{z}+\Upsilon_{z}\right),-i \hbar \partial_{\varphi}-e A\right]
\end{gathered}
$$

Indeed, we have $\left[\hat{H}, \partial_{\varphi}\right]=0$.

By calculation similar to (3.12)-(3.17), we find $F_{\theta}=0$, the second commutator $\hat{T}$ also has the same form of (3.16),

$$
\hat{T}=i K\left(\vartheta^{0 r z} \hat{P}_{z}+\vartheta^{0 r \varphi} \hat{P}_{\varphi}\right)
$$

where $K=K(r, z)$ is a smooth real function to be determined. Substituting (3.22) and (3.24) into (3.9), we get the integrable conditions as follows:

$1^{\circ} R=R(r), K=Z=Z(r)$. By a transformation $d r^{\prime}=R(r) d r$, then in equivalent sense we have

$$
R=1, \quad K=Z=Z(r)
$$

where $Z(r)$ is any given smooth function.

$2^{\circ}$ If $\rho=Z(r) f(z)$ and $f(z)$ is any given smooth function, we have conditions for other parameters,

$$
\partial_{z} A_{0}=\partial_{z} F_{\gamma}=\partial_{r} A=0 .
$$


This is similar to (3.17) in spherical coordinate system.

$3^{\circ}$ In the general case of $\rho \neq Z(r) f(z)$, assuming $\partial_{\varphi} \phi=i m_{z} \phi$, we have

$$
\partial_{z} A_{0}=\partial_{z} F_{\gamma}=0, \quad A=\hbar m_{z}+\frac{\rho Q(z)}{Z}
$$

where $Q(z)$ is any given arbitrary functions.

Especially, in cylindrical coordinate system we have

$$
R=K=Z=1, \quad \rho=r, \quad \Upsilon_{\mu}=\left(0, \frac{1}{2 r}, 0,0\right)
$$

and integrable condition becomes

$$
A_{0}=V(r), \quad e A=r Q(z)+\hbar m_{z}, \quad \partial_{z} F_{\gamma}=0
$$

In Minkowski space-time, the condition for vector potential becomes

$$
e \vec{A}=e A_{\varphi} \vartheta^{\varphi}=\left(Q(z)+\frac{\hbar m_{z}}{r}\right)(-\sin \varphi, \cos \varphi, 0)
$$

which explicitly depends on $m_{z}$. So the integrable condition is state dependent. In this case, we have

$$
\hat{T}=i \vartheta^{012}\left(-i \hbar \partial_{z}\right)-i \vartheta^{013} Q(z)
$$

If $Q=\frac{1}{2}(2 n-1) \hbar z^{-1}$, the eigen function of $\hat{T} \phi=\lambda_{z} \hbar \phi$ can be expressed by Bessel functions

$$
\begin{gathered}
\phi=\left[u(r)+v(r) \vartheta^{5}\right](a, a, b,-b)^{T} \\
a=\sqrt{|z|} J_{n}\left(\left|\lambda_{z}\right| z\right), \quad b=\frac{i}{\lambda_{z} \sqrt{|z|}}\left[\left|\lambda_{z} z\right| J_{n+1}\left(\left|\lambda_{z}\right| z\right)-2 n J_{n}\left(\left|\lambda_{z}\right| z\right)\right] .
\end{gathered}
$$

In which $\lambda_{z}$ is determined by boundary condition and $(u, v, E)$ by $\hat{H} \phi=E \phi$.

\section{INTEGRABLE CONDITIONS FOR PAULI EQUATION}

From the calculation of the above section, we find that there are too many constraints for Dirac equation, one can hardly get exact solution for complicated potential. But for the Pauli and Schrödinger equations, the situation is much better. We consider the non-relativistic approximation,

$$
i \hbar \partial_{t} \phi=\hat{H} \phi, \quad \hat{H}=\frac{\hbar^{2}}{2 m}(\sigma \cdot \hat{p})^{2}+e V .
$$


Where $\sigma_{k}$ forms the generators of Clifford algebra $C \ell_{3,0}$. Substituting $\hat{p}=-i \hbar \nabla-e \vec{A}$ into $(4.34)$, omitting $\mathrm{O}\left(\vec{A}^{2}\right)$ term and using the Coulomb's gauge condition $\nabla \cdot \vec{A}=0$, we get

$$
\begin{aligned}
\hat{H} & =-\frac{\hbar^{2}}{2 m} \Delta+e V+\frac{i e \hbar}{2 m}\left(\partial_{k} A_{l}-\partial_{l} A_{k}\right) \sigma^{k l} \\
& =-\frac{\hbar^{2}}{2 m} \Delta+e V+\frac{i e \hbar}{2 m} B^{m}\left(\epsilon_{k l m} \sigma^{k l}\right) \\
& =-\frac{\hbar^{2}}{2 m} \Delta+e V-\frac{e \hbar}{2 m} B^{k} \sigma_{k} .
\end{aligned}
$$

As an example to show the standard procedure of Clifford algebra, we derive the equation of magnetostatics. In $C \ell_{3,0}$, the generators or frame $\sigma_{a}(a \in\{1,2,3\})$ meet algebraic rules

$$
\sigma_{a} \sigma_{b}+\sigma_{b} \sigma_{a}=2 \delta_{a b}, \quad \sigma_{\mu} \sigma_{\nu}+\sigma_{\nu} \sigma_{\mu}=2 g_{\mu \nu}, \quad \sigma^{a}=\delta^{a b} \sigma_{b}
$$

We have the basis

$$
I \in \Lambda^{0}, \quad \sigma_{a} \in \Lambda^{1}, \quad \sigma_{a b} \equiv \sigma_{a} \wedge \sigma_{b}=i \epsilon_{a b c} \sigma^{c} \in \Lambda^{2}, \quad \sigma_{a b c}=i \epsilon_{a b c} I \in \Lambda^{3}
$$

They correspond to scalar, vector, pseudo vector and pseudo scalar respectively. Denoting

$$
\mathbf{D}=\sigma^{\alpha} \nabla_{\alpha}, \quad \mathbf{A}=\sigma^{\beta} A_{\beta}, \quad \mathbf{B}=\sigma^{\beta} B_{\beta}, \quad F_{\mu \nu}=\partial_{\mu} A_{\nu}-\partial_{\nu} A_{\mu}
$$

then we have

$$
\begin{aligned}
\mathbf{D A} & =\sigma^{\alpha} \sigma^{\beta} \nabla_{\alpha} A_{\beta}=\left(g^{\alpha \beta}+\sigma^{\alpha \beta}\right) \nabla_{\alpha} A_{\beta} \\
& =\nabla_{\mu} A^{\mu}+\frac{1}{2} \sigma^{\mu \nu}\left(\partial_{\mu} A_{\nu}-\partial_{\nu} A_{\mu}\right)=A_{; \mu}^{\mu}+\frac{1}{2} \sigma^{\mu \nu} F_{\mu \nu}
\end{aligned}
$$

Clearly DA $\in \Lambda^{0} \cup \Lambda^{2} \subset C \ell_{3,0}$. By Thm.2, we get

$$
\begin{aligned}
\mathbf{D}^{2} \mathbf{A} & =\sigma^{\alpha} \partial_{\alpha} A_{; \mu}^{\mu}+\frac{1}{2} \sigma^{\alpha} \sigma^{\mu \nu} \nabla_{\alpha} F_{\mu \nu} \\
& =\sigma^{\alpha} \partial_{\alpha} A_{; \mu}^{\mu}+g^{\alpha \mu} \sigma^{\nu} \nabla_{\alpha} F_{\mu \nu}+\frac{1}{2} \sigma^{\alpha \mu \nu} \nabla_{\alpha} F_{\mu \nu} \\
& =\sigma_{\alpha}\left(\partial^{\alpha} A_{; \mu}^{\mu}-\nabla_{\mu} F^{\alpha \mu}\right)+\frac{\sigma^{123}}{2 \sqrt{g}}\left(\epsilon^{\alpha \mu \nu} \nabla_{\alpha} F_{\mu \nu}\right)
\end{aligned}
$$

Therefore $\mathbf{D}^{2} \mathbf{A} \in \Lambda^{1} \cup \Lambda^{3}$, and then $\mathbf{D}^{3} \mathbf{A} \in \Lambda^{0} \cup \Lambda^{2}$. If the $\sigma^{123}=i$ term does not vanish in $\mathbf{D}^{2} \mathbf{A}$, the dynamical equation should be closed at least in $\mathbf{D}^{4} \mathbf{A}$. However, all field equations in physics should be closed in $\mathbf{D}^{2}$, therefore we have

$$
\mathbf{D}^{2} \mathbf{A}=-b^{2} \mathbf{A}+e \mathbf{J}=\sigma_{\alpha}\left(-b^{2} A^{\alpha}+e J^{\alpha}\right)
$$

In contrast the above equation with (4.39), we get the first order dynamical equation as

$$
\partial^{\mu} A_{; \mu}^{\mu}-\nabla_{\nu} F^{\mu \nu}+b^{2} A^{\mu}=e J^{\mu}, \quad \epsilon^{\alpha \mu \nu} \nabla_{\alpha} F_{\mu \nu}=0
$$


In which all variables are covariant ones with clear physical meanings. If Coulomb's gauge condition $A_{; \mu}^{\mu}=0$ holds, for long distance interaction $b=0,(4.41)$ gives the complete Maxwell equation system for vector potential $\mathbf{A}$ in curved space-time,

$$
-\Delta A^{\mu}=\epsilon^{\mu \alpha \beta} B_{\alpha ; \beta}=e J^{\mu}, \quad B_{; \mu}^{\mu}=0, \quad B^{\alpha}=\epsilon^{\alpha \mu \nu} F_{\mu \nu}
$$

Now we examine the axisymmetric potential in spherical coordinate system $(r, \theta, \varphi)$. In this case we have

$$
f_{\mu}{ }^{a}=\operatorname{diag}(1, r, r \sin \theta), \quad \sqrt{g}=r^{2} \sin \theta, \quad V=V(r, \theta), \quad \vec{A}=\vec{A}(r, \theta)
$$

By $B_{; \mu}^{\mu}=0$, we get

$$
\left(\partial_{r}+\frac{2}{r}\right) B^{r}+\left(\partial_{\theta}+\cot \theta\right) B^{\theta}=0
$$

Since the magnetic field B in (4.35) is given, we need not express it in covariant form, otherwise the Pauli equation (4.35) becomes complicated. Assume B is axisymmetric, then we have

$$
\vec{B}=\left(B_{\rho} \cos \varphi, B_{\rho} \sin \varphi, B_{z}\right), \quad B^{k} \sigma_{k}=\left(\begin{array}{cc}
B_{z} & B_{\rho} e^{-i \varphi} \\
B_{\rho} e^{i \varphi} & -B_{z}
\end{array}\right)
$$

in which $B_{\rho}$ and $B_{z}$ are functions of $(r, \theta) . B^{k} \sigma_{k}$ depends on $\varphi$ is troublesome, we make a transformation

$$
\phi=\left(\begin{array}{cc}
0 & \exp \left(-\frac{i \varphi}{2}\right) \\
\exp \left(\frac{i \varphi}{2}\right) & 0
\end{array}\right) \psi
$$

then we get the Pauli equation and Hamiltonian in spherical coordinate system as

$$
\begin{aligned}
i \partial_{t} \psi & =\hat{H} \psi, \quad \hat{H}=\frac{\hbar^{2}}{2 m}\left(-\Delta+\frac{1}{4 r^{2} \sin ^{2} \theta}\right)+e V-\frac{e \hbar}{2 m}\left(B_{\rho} \sigma_{1}+B_{z} \sigma_{3}\right), \\
\Delta & =\left(\partial_{r}^{2}+\frac{2}{r} \partial_{r}\right)-\frac{1}{r^{2}} \hat{L}^{2}, \quad \hat{L}^{2}=-\left(\partial_{\theta}^{2}+\cot \theta \partial_{\theta}+\frac{1}{\sin ^{2} \theta} \partial_{\varphi}^{2}\right),
\end{aligned}
$$

where $\hat{L}$ is the angular momentum operator and $\hat{L}_{z}=-i \partial_{\varphi}$.

Obviously, for axisymmetric potential, we have

$$
\left[\hat{H}, \hat{L}_{z}\right]=0, \quad \partial_{\varphi} \leftrightarrow m_{z} i
$$

where $m_{z}$ is an integer. We look for the following second order Hermitian operator

$$
\hat{T}\left(\partial_{\theta}, \partial_{\varphi}\right)=-\left(\hat{L}^{2}+\frac{1}{4 \sin ^{2} \theta}\right)+T^{0}-i T^{\theta}\left(\partial_{\theta}+\frac{1}{2} \cot \theta\right)-i T^{\varphi} \partial_{\varphi}
$$


By Hermitian of $\hat{T}$, we have

$$
T^{0}=X+Y_{a} \sigma^{a}, \quad T^{\theta}=P+Q_{a} \sigma^{a}, \quad T^{\varphi}=R+Z_{a} \sigma^{a} .
$$

By $\left[\hat{T}, i \partial_{\varphi}\right]=0$, we find that all coefficients are independent of $\varphi$. Then we get integrable condition as

$$
[\hat{H}, \hat{T}]=[\hat{H}, \hat{T}]_{A}-\frac{\hbar^{2}}{2 m} \Delta \hat{T}+\hat{L}^{2} \hat{H}+i T^{\theta} \partial_{\theta} \hat{H}=0
$$

in which $[\hat{H}, \hat{T}]_{A}$ is commutative operation taking differential operators as ordinary number. By straightforward calculation, we get $T^{\theta}=T^{\varphi}=Y_{2}=0$ and

$$
\begin{aligned}
\Delta X & =-\frac{2 m e}{\hbar^{2}}\left(\partial_{\theta}^{2} V+\cot \theta \partial_{\theta} V\right), \\
Y_{1} B_{z}=Y_{3} B_{\rho}, \quad \Delta Y_{1} & =\frac{e}{\hbar}\left(\partial_{\theta}^{2} B_{\rho}+\cot \theta \partial_{\theta} B_{\rho}\right), \quad \Delta Y_{3}=\frac{e}{\hbar}\left(\partial_{\theta}^{2} B_{z}+\cot \theta \partial_{\theta} B_{z}\right) .
\end{aligned}
$$

(4.44) or $\nabla \cdot \vec{B}=0$ becomes

$$
\left(r \sin \theta \partial_{r}+\cos \theta \partial_{\theta}+\frac{1}{\sin \theta}\right) B_{\rho}+\left(r \cos \theta \partial_{r}-\sin \theta \partial_{\theta}\right) B_{z}=0
$$

With natural boundary condition, (4.53) can be easily solved. For example, we have

$$
\begin{array}{rlrl}
V & =\Phi(r)+\frac{1}{r^{2}} U(\theta), & X & =-\frac{2 m e}{\hbar^{2}} U(\theta) ; \\
V=\Phi(r)+\frac{\kappa}{r^{3}} \cos ^{2} \theta, & X & =-\frac{2 m e \kappa}{\hbar^{2} r} \cos ^{2} \theta ; \\
V=\Phi(r)+\frac{\kappa}{r^{3}} \sin ^{2} \theta \cos ^{2} \theta, & X & =-\frac{2 m e \kappa}{3 \hbar^{2} r} \sin ^{2} \theta \cos ^{2} \theta .
\end{array}
$$

$X$ is used to construct a commutator, we only need to choose the simplest particular solution. The above potentials are more coincident to the practical situation inside an atom than $V(r)$, where the charge distribution is similar to an ellipsoid.

$\hat{T}$ is a linear operator, and (4.53) is a linear equation for $X$ and $V$, so the solutions satisfy superposition principle. If the magnetic field can be omitted, for potentials with even degree terms of $\sin ^{2 k} \theta$ and $\cos ^{2 k} \theta$, such as (4.57) and (4.58), the eigen solution to $\hat{T} \psi=-J \psi$ can be easily solved. For example, we have solution for potential (4.57),

$$
\begin{gathered}
\left(\partial_{\theta}^{2} \psi+\cot \theta \partial_{\theta}-\frac{n^{2}}{\sin ^{2} \theta}-\frac{2 m e \kappa}{\hbar^{2} r} \cos ^{2} \theta\right) \psi_{\theta}=-J \psi_{\theta}, \quad n=m_{z} \text { or } \sqrt{m_{z}^{2}+\frac{1}{4}}, \\
\psi_{\theta}=\sin ^{n} \theta\left(C_{1} \cos \theta F\left(0, \frac{1}{2}, n, \frac{m e \kappa}{2 \hbar^{2} r}, j, \cos ^{2} \theta\right)+C_{2} F\left(0,-\frac{1}{2}, n, \frac{m e \kappa}{2 \hbar^{2} r}, j, \cos ^{2} \theta\right)\right),
\end{gathered}
$$

in which $j=\frac{1}{4}\left(n^{2}+1-J\right), F(\cdot)$ is Heun's confluent function. The total eigen function takes the form $\psi=f(r) \psi_{\theta}(r, \theta) e^{i m_{z} \varphi}$, where $f(r)$ can be determined by radial eigen equation of (4.47). The eigen function $\psi_{\theta}$ depends on both $(r, \theta)$, so the method is a generalized separation of variables. 
Different from the arbitrary scalar potential $V$, the magnetic field $\vec{B}$ and $\vec{Y}$ are constrained by (4.54) and (4.55). Making transformation of variables

$$
\begin{aligned}
& Y_{1}=K B_{\rho}, \quad B_{\rho}=-\cos \theta \partial_{\rho} A+\frac{1}{r} \sin \theta \partial_{\theta} A \\
& Y_{3}=K B_{z}, \quad B_{z}=\sin \theta \partial_{r} A+\frac{1}{r} \cos \theta A+\frac{1}{r \sin \theta} A
\end{aligned}
$$

substituting it into (4.54) we get two equations for $(K, A)$. The equation is quite complicated, which means only in some special magnetic field the Pauli equation is integrable. Here the vector potential $A$ is defined as

$$
\mathbf{A}=A_{\varphi} \sigma^{\varphi}=A(-\sin \varphi, \cos \varphi, 0), \quad A_{\varphi}=f_{\varphi}^{a} A_{a}=r \sin \theta A
$$

The simplest case is constant magnetic field

$$
B_{\rho}=0, \quad B_{z}=B, \quad K=1, \quad A=\frac{1}{2} B r \sin \theta
$$

In this case one component of $\psi$ vanishes, and Pauli equation (4.47) reduces to Schrödinger equation. The more complicated but important case is the magnetic field of dipole. Assume $M \sigma_{3}$ is a magnetic dipole at the origin, we have solution for (4.54) and (4.55),

$$
A=\frac{\mu_{0} M}{4 \pi r^{2}} \sin \theta, \quad K=\frac{e}{\hbar} r^{2}, \quad B_{\rho}=\frac{3 \mu_{0} M}{4 \pi r^{3}} \sin \theta \cos \theta, \quad B_{z}=\frac{\mu_{0} M}{4 \pi r^{3}}\left(3 \cos ^{2} \theta-1\right) .
$$

In this case, we have

$$
\begin{aligned}
& \hat{T}=-\left(\hat{L}^{2}+\frac{1}{4 \sin ^{2} \theta}\right)+X+S \\
& S=Y_{1} \sigma_{1}+Y_{2} \sigma_{2}=\frac{e}{\hbar} r^{2}\left(B_{\rho} \sigma_{1}+B_{z} \sigma_{3}\right)
\end{aligned}
$$

To solve the eigen equation $\hat{T} \psi=-J \psi$, we have to diagonalize the matrix $S(4.67)$ in $\hat{T}$. In the language of Clifford algebra, we should make a rotational transformation for vector $S$ around $y$-axis[18]. The algebra operation is given by $\psi=\left(p+q \sigma_{31}\right) \phi$ and the diagalized operator has the following form,

$$
\left(p-q \sigma_{31}\right) \hat{T}\left(p+q \sigma_{31}\right)=\left(p^{2}+q^{2}\right)\left(-\hat{L}^{2}-\frac{1}{4 \sin ^{2} \theta}+X\right)+P+Q \sigma_{3}
$$

where $p, q, P, Q$ are all real functions.

By (4.68) we get the equations for $p, q$ as

$$
\begin{array}{r}
3 \cos \theta \sin \theta\left(p^{2}-q^{2}\right)+2\left(3 \cos ^{2} \theta-1\right) p q=0, \\
q\left(\partial_{\theta}^{2} p+\cot \theta \partial_{\theta} p\right)-p\left(\partial_{\theta}^{2} q+\cot \theta \partial_{\theta} q\right)=0 .
\end{array}
$$


The solutions are given by

$$
\begin{aligned}
& p=\sqrt{\frac{\sin \theta\left(\sqrt{3 \cos ^{2} \theta+1}+1\right) \sqrt{3 \cos ^{2} \theta+1}}{\left(2+\sqrt{3 \cos ^{2} \theta+1}\right)\left(\cos ^{2} \theta+1\right)}}, \\
& q=\sqrt{\frac{\sin \theta\left(\sqrt{3 \cos ^{2} \theta+1}-1\right) \sqrt{3 \cos ^{2} \theta+1}}{\left(2-\sqrt{3 \cos ^{2} \theta+1}\right)\left(\cos ^{2} \theta+1\right)}}, \\
& P=-\frac{27 \cos ^{8} \theta-180 \cos ^{6} \theta+38 \cos ^{4} \theta+52 \cos ^{2} \theta-1}{6 \sin ^{3} \theta\left(3 \cos ^{2} \theta+1\right)\left(\cos ^{2} \theta+1\right)^{3}}, \\
& Q=\frac{2 e M}{3 \hbar r} \frac{\sqrt{\left(3 \cos ^{2} \theta+1\right)^{3}}}{\sin \theta\left(\cos ^{2} \theta+1\right)}, \quad p^{2}+q^{2}=\frac{2\left(3 \cos ^{2} \theta+1\right)}{3 \sin \theta\left(\cos ^{2} \theta+1\right)} .
\end{aligned}
$$

It is interesting that $(p, q)$ and $P$ are independent of $r$ and $M$, so they only depend on the shape of magnetic force line. Under this transformation, the Hamiltonian $\hat{H}$ in the original equation (4.47) is also diagonalized simultaneously.

Under the above transformation, the operator is diagonalized and one of the component of spinor $\phi$ vanishes. We get the following eigen equation for a scalar field $\phi_{\downarrow}$ or $\phi_{\uparrow}$ as

$$
\left(-\hat{L}^{2}-\frac{1}{4 \sin ^{2} \theta}+X+\frac{P \pm Q}{p^{2}+q^{2}}\right) \phi=-J \phi .
$$

Substituting (4.71)-(4.74) into the above equation we get

$$
\begin{aligned}
\left(\partial_{\theta}^{2}+\cot \theta \partial_{\theta}\right) \phi-\left(\frac{m^{2}}{\sin ^{2} \theta} \pm \frac{e M}{\hbar r} \sqrt{3 \cos ^{2} \theta+1}\right) \phi+X \phi+ & \\
+\frac{3 \cos ^{2} \theta\left(3 \cos ^{4} \theta-10 \cos ^{2} \theta-5\right)}{\left(3 \cos ^{2} \theta+1\right)^{2}\left(\cos ^{2} \theta+1\right)^{2}} \phi & =-J \phi .
\end{aligned}
$$

Then the solution of (4.47) is given by

$$
\psi=\left(p+q \sigma_{31}\right) \phi=\left(\begin{array}{c}
p \\
-q
\end{array}\right) \phi_{\uparrow}, \quad \text { or }\left(\begin{array}{l}
q \\
p
\end{array}\right) \phi_{\downarrow}
$$

The rigorous eigen solution to (4.76) may be integrable, because the solution $\psi$ to the original equation (4.47) can be exactly derived if $\mathbf{B}=0$ and $V=V(r)$. In this case we have solution for (4.76) as

$$
\phi=\frac{p \pm q}{p^{2}+q^{2}} \psi
$$

The general solution of (4.76) should also take this form, and $\psi$ should be a hypergeometric function. However, the computation is very complicated. 


\section{DISCUSSION AND CONCLUSION}

From the above discussion we learn that, physical variables expressed by Clifford algebra simultaneously display tetrad and parameters as shown in (3.30). Clifford algebra automatically arrange a large amount of information in order, and make parameters and relations with clear physical meanings. The representation in Clifford algebra has a neat form with no more or less contents, which is also valid in curved space-time. The second commutator $\hat{T}$ of Dirac equation in (3.16) and (3.24) is simply a covariant operator. For Pauli and Schrödinger equations, Many solutions in axisymmetric potential $V(r, \theta)$ and magnetic field $\mathbf{B}(r, \theta)$ are also integrable. We get the specific scalar equation with dipole magnetic field. In this paper, the integrable condition is only regarded as an algebra condition, which is unnecessarily related to some complicated geometric symmetry. By the insights of Clifford algebra, the physical researches may be greatly promoted.

[1] W. Greiner, B Müller, Quantum Mechanics-Symmetries, Ch.3, Springer-Verlag Berlin Heidelberg, 2001

[2] Y. Q. Gu, Integrable conditions for Dirac Equation and Schrödinger equation, arXiv:0802.1958.

[3] W. Greiner, Relativistic Quantum Mechanics-Wave Equations, Springer-Verlag Berlin Heidelberg, 1990

[4] V. G. Bagrov, M. C. Baldiotti, D. M. Gitman, I. V. Shirokov, New solutions of relativistic wave equations in magnetic fields and longitudinal fields, J. Math. Phys. 43 (2002) 2284-2305, hep-th/0110037

[5] V. M. Villalba, The angular momentum operator in the Dirac equation, CPT-94/P.3027, hepth/9405033

[6] X. Y. Gu, Z. Q. Ma, S. H. Dong, Exact solutions to the Dirac equation for a Coulomb potential in D +1 dimensions, Int. J. Mod. Phys. E, 2002, 11(04):335-346.

[7] X.Y. Gu, Z.Q. Ma, S.H Dong, The Levinson theorem for the Dirac equation in D+1 dimensions. Phys. Rev. A 67(6), 062715 (2003)

[8] S. H. Dong, J. García-Ravelo, Exact solutions of the s-wave Schrödinger equation with Manning-Rosen potential, Phys. Scr. 75 (2007) 307-309

[9] D. Hestenes, G. E. Sobczyk, Clifford Algebra to Geometric Calculus. A Unified Language for Mathematics and Physics, Amer. J. Phys. 53(5), 510-511(1984)

[10] D. Hestenes, A Unified Language for Mathematics and Physics, Adv. Appl. Clifford Algebras 1(1): $1-23(1986)$

[11] J. Keller, Spinors and multivectors as a unified tool for spacetime geometry and for elementary particle physics, Int. J. Theor. Phys. 30(2): 137-184 (1991).

[12] J. Lasenby, A. N. Lasenby, C. J. L. Doran, A unified mathematical language for physics and engineering in the 21st century, Phil. Trans. Roy. Soc. 358.1765: 21-39(2000). 
[13] Y. Q. Gu, Clifford Algebra, Lorentz Transformation and Unified Field Theory, Adv. Appl. Clifford Algebras 28(2):37, (2018)

[14] Y. Q. Gu, Space-Time Geometry and Some Applications of Clifford Algebra in Physics, Adv. Appl. Clifford Algebras 28: 79, (2018).

[15] P. Lounesto, Clifford Algebras and Spinors, Cambridge Univ. Press, Cambridge 2001.

[16] D. S. Shirokov, Clifford algebras and their applications to Lie groups and spinors, Proceedings of the Nineteenth International Conference on Geometry, Integrability and Quantization, Ivailo M. Mladenov and Akira Yoshioka, eds. (Sofia: Avangard Prima, 2018), 11-53, arXiv:1709.06608

[17] Y. Q. Gu, A Note on the Representation of Clifford Algebra, Preprints 2020, 2020020466 (doi:10.20944/preprints202002.0466.v1).

[18] Y. Q. Gu, Some Applications of Clifford Algebra in Geometry, Preprints 2020, 2020020140 (doi:10.20944/preprints202002.0140.v1)

[19] J. M. Nester, Special orthonormal frames, J. Math. Phys. 33, 910 (1992).

[20] Y. Q. Gu, The Simplification of Spinor Connection and Classical Approximation, arXiv:gr-qc/0610001

[21] Y. Q. Gu, Natural Coordinate System in Curved Space-time, J. Geom. Symmetry Phys. 47 (2018) 51-62, arXiv:gr-qc/0612176, doi: 10.7546/jgsp-47-2018-51-62

[22] Y. Q. Gu, The Quaternion Structure of Space-time and Arrow of Time, J. Mod. Phys. V3, 570$580(2012)$. 